\title{
Tissue-Centered Dynamics are Superimposed on Cellular Transitions in Co-operative Tumorigenesis and spread
}

\author{
*Lawrence M Agius \\ Department of pathology, University of Malta Medical School, Europe
}

Submission: April 23, 2017; Published: June 26, 2017

"Correspondence author: Lawrence M Agius, Department Of Pathology, University of Malta Medical School, Mater Dei Hospital, Tal-Qroqq, Msida, 27 "ballarat" guzeppe Caruana Street, tal-virtu, rabat, rbt09 Malta, Europe, Tel: 356-21451752; Email: lawrence.agius@um.edu.mt

Abstract

Transforming events of cells and tissues in tumorigenesis promote the defining indices of cellular proliferation and also of the microenvironmental remodeling as malignant cells evolve from epithelial to mesenchymal components. It is within systems of progression that permissiveness allows for the repeated re-establishment of paracrine and autocrine events involving cytokine and chemokine events that lead particularly to systemic propagation of dynamic turnover. It is indeed necessary to recognize stromal multipotentiality as central attribute characterization of the malignant cells themselves in defining system sub-sets in genomic instability and of disordered gene expression profiles. Modulation of such gene expression is inductive phenomena of acquisition in primarily tissue-based incorporation of proliferative and spreading attributes.

\section{Introduction}

The constitutional and reactive potential attributes of cancer-associated stroma include a full range of proliferative and synthetic mechanistic pathways that may include in particular an epithelial-mesenchymal transition series of phenomena. Immune cells and immunomodulatory markers may potentially be targeted therapeutically [1]. Tumor-associated macrophages are associated with invasion and migration of several cancer types [2]. It is the directional attribute especially of the marrowderived circulating mesenchymal cells that is permissive in inducing the establishment of carcinoma-associated fibroblasts and of myofibroblasts within the tumor-associated stroma.

\section{Reactive Stroma}

The dimensions of the reactive stroma in cancer include the evolutional attributes of a heterogeneous population of cells centered on the vascular-derived cell and matrix components of the vascular wall, especially of the post-capillary venules within the cancer-associated stroma. The proliferative phenotypes of these stromal cell populations appear implicated directly in the dynamics of the epithelial-mesenchymal transitions in tumors. Micro-environmental gradients comprise hypoxia, $\mathrm{pH}$, ions, and chemo tactic induction, besides concentration, stiffness and organization modifications of the extracellular matrix [3]. It is within the remodeling of the tumor-associated stroma that dynamics of cell turnover further exacerbate the dual persistence of epithelial and subsequently of the carcinoma associated fibroblasts and myofibroblasts in cases of established metastatic potential and progression. Local and systemic tumor environment attributes, especially immunoscore, budding, tumor stroma percentage are stage-independent determinants of survival in operable colon cancer [4]. Decreased tumorcell susceptibility to natural-killer mediated lysis is triggered by anti-tumor immune effects exerted by cancer-associated fibroblasts [5].

\section{Cell Transitions}

Integral co-operative changes in epithelial-mesenchymal transition indicate a central role for transforming growth factorbeta in a manner that involves immune suppressive mechanisms on the one hand and of progressive transitions of stroma that initially is reactive and subsequently conducive towards further growth of the neoplasm. The nature of epithelial-mesenchymal transitions accounts for the evolutionary course of a neoplasm that is integrally incorporated with stroma. Intratumoral intravasation by cancer cells is independent of invasion of the tutor stroma [6]. The heterogeneous cell populations within 


\section{Cancer therapy \& Oncology International Journal}

the tumor micro-environment account directly to an enhancing series of mechanisms that promote the establishment of recurrent and metastatic disease.

Cancer-associated fibroblasts promote migration and group of malignant cells in a Hepatocyte Growth Factor-dependent manner and co targeting of cancer cells and stroll cells is necessary to prevent therapeutic resistance [7]. Beyond such proliferative and pro-fibrotic activities there evolves the dimensional propensity for cell-cell communication between carcinomatous cells and a micro-environment that proves progressively more and more dynamic in proliferative and vascular activity. Wnt16 is a key factor leading to cisplatin resistance in malignant tumors and achieves a synergist antitumor effect in stoma-rich bladder carcinoma model when delivered as nanoparticles [8].

\section{Promotional Dynamics}

Promotional dynamics and the permissive phenomena in carcinogenesis account for the emergence of malignant cells that further contributes to stromal participation in tumorigenesis and growth of the neoplasm. Transforming potential invokes paracrine processes that are centrally implicated in further creating epithelial-mesenchymal transitions. The myofibroblasts prove prototypes in such induction of systems of co-operative dimension. Clearly, the permissive elements of transforming potential is a genome-based series of adaptive sets of cellular expression as further evidenced by the subsequent emergence of metastatic attributes of the tumor cells. The micro-environment of tumors is related to tumor prognosis as shown by periostin expression by cancer-associated fibroblasts [9].

Angiogenesis and stem cells account for the established genetic profiles of malignant cells in a manner that implicates the transforming dynamics of the cancer-associated fibroblasts and of myofibroblasts as systems of amplifying potential. There is increasing evidence for a prognostic role of hypoxia in highgrade tissue sarcomas [10]. The realization for progression of tumor growth incorporates a persistence of stroma that is locally projected as emerging neoplastic cell proliferative activities. In vitro assays show that urokinase plasminogen activator promotes oesophageal squamous carcinoma cell proliferation, migration and infiltration via PI3K/AKT and ERK signaling pathways [11].

\section{Transformation}

Basic considerations of transforming phenomena are integral to the further compound participation of cell injury in the face of responding homeostatic mechanisms. Drugs can prime the tumor stroma to enhance chemotherapy [12]. Homeostatic elements are profiles for further expansion of the vascular components that extend the focality of malignant transformation towards directional creation of the epithelialmesenchymal transition. Asporin, an extracellular matrix protein, enhances epithelial-mesenchymal transition, invasion and migration of pancreatic cancer cells [13]. It is in view of dimensional reconstitution of a non-healing wound that neoplasia is itself a product of paracrine secretion and induction for further growth and spread of the malignant cells. Tumorreactive storm acts as a paramount tumor-promoting drive in biliary carcinogenesis and myofibroblasts, macrophages, endothelial cells and mesenchymal stem cells continuously reactive malignancy via cytokines, chemokine, growth factors, morphogens and proteinases [14].

\section{Reparative Process}

Participating indices of a reparative process are thus translated to the further profile progressions that incorporate dual systems within the phenomenal emergence of the epithelial-mesenchymal transition. It is within the defining cooperative milieu of the cancer-associated stroma that heterogeneous cell populations incorporate the potentiality for further transforming attributes between various elements of cell and interacting matrix.

Tumor biology as a primarily inductive phenomenon is selfprogressive and leads to the formulation of transition within the stroma itself. It is clearly in the defining of biologic transitions that the stromal micro-environment promotes the accumulation of cellular elements that interact with extracellular matrix. Facilitated gene expression incorporates a transforming body of profile indices that relate the amount of stroma to tumorigenesis and spread of the neoplasm. The vascular endothelial cells are cooperative indices and prove indices for further tumor growth in terms of oxidative stress and enzymatically-mediated processes of tissue remodeling.

\section{Pathway Interactivity}

Realized pathways of anaerobic metabolic pathways and the matrix degradation by such enzymes as metalloproteases allow for permissive emergence of proliferation of both malignant cells and stromal cell elements. Metalloproteases are dynamic singling proteases of cancer and are modulated by stroll cellular heterogeneity, extracellular matrix, tissue oxygenation and inflammation [15]. Transforming Growth Factor Beta-1 signaling increases motility of diffuse gastric carcinoma via expression of Rhomboid 5 Homolog 2 and induce invasion by gastric cancer cells [16]. It is further to such defined indices of progression of transforming growth factor-beta and fibroblast activation protein that there is established recurrent dynamics of epithelialmesenchymal and mesenchymal-epithelial transitions within the aberrant homeostatic milieu of the tumor-associated stroma. The fact that pancreatic adenocarcinomas rely on embedded super enhance transcriptional mechanisms may render clusters of multiple enhancers susceptible to targeted therapies [17].

\section{Shift Formulation}

Periodic shifts in formulating cellular identity profiles extend to include stem cell sub-sets within a micro-environmental 


\section{Cancer therapy \& Oncology International Journal}

milieu of evolving multi-potentiality. The incremental profiles of stromal cell constituents allows for modulation that is strictly inductive between cell types and within sub-sets of progenitor cells of origin of the neoplasm. Distinct and intersecting molecular profiles define cancer-associated fibroblast activation states and hierarchical cellular heterogeneity and are potential therapeutic targets [18].

\section{Tissue-Based Dynamics}

A tissue-based profile of attribute-determination contrasts with the conventional cell-centered dimensions of tumorigenesis. It is the defined dimensions of an epithelial-stromal series of transitions that the co-operative dualism of tumor and its stroma permits the emergence of active attributes of tumor cell proliferation and spread. Tissue-specific attributes transcend the restrictive attributes of dimensional tumorigenesis beyond the transforming phenomena of individual components of a non-healing wound. It is particularly in terms of coordinated permissive and transforming dimensions of secreted autocrine and paracrine elements that cytokines and chemokines help segregate in quasi-identifiable terms the malignant cells and the remodeling tumor-associated micro-environment. In real terms, ongoing processes collaborate with established elements of transforming identity and allow for permissive profiles of identified profiles of a reactive stroma that further emerges as cancer-associated fibroblasts and myofibroblasts.

Micro-RNAs that are expressed in cancer-associated fibroblasts may mediate regulation cancer progression and spread, cancer metabolism, stem cell attributes and chemo resistance [19]. Contrast dynamics are defining indices of a transitional permissiveness that provokes the further promotion of tissue profile in terms of epithelial-mesenchymal transition. Substantial co-evolution of malignant cells and of stroma is centered on a perivascular niche for stem cells in providing for the dynamics of a malignant transformation event.

\section{Conclusion}

Cellular transformation as epithelial-mesenchymal and mesenchymal-epithelial transitions is profile-determinant in the permissively inherent system for tumorigenesis in a manner that characterizes and re-characterizes outcome of damage as a primarily tissue-based phenomenon of progression. It is within the defining terms of a tumor-associated milieu that homeostatic mechanisms are adaptive processes on the one hand and further incremental participants in the malignant transformation process of self-renewal. It is further to the realized process of cellular transformations that the neoplasm incorporates attributes of adaptation in its own right and as further characterized by dynamics of metastatic spread. Increments of co-operative extension of epithelial-stromal transitions in particular are profile determinants in established identity for a primarily tissue lesion that is proliferative and also genomically unstable.

\section{References}

1. Dongre A, Rashidan M, Reinhardt F, Bagnato A, Keckesova Z, et al. (2017) "Epithelial-to-mesenchymal transition contributes to immunosuppression in breast carcinomas" Cancer Res pii: canres 3292.2016.

2. Zhao X, Qu J, Sun Y, Wang J, Liu X, et al. (2017) Prognostic significance of tumor-associated macrophages in breast cancer: a meta-analysis of the literature. Oncotarget 8(18): 30576-30586.

3. Odin MJ, Weaver VM (2017) Physical and chemical gradients in the tutor microenvironment regulate tumor cell invasion, migration, and metastasis. Cold Spring Harb Symp Quant Biol pii: 030817.

4. Park JH, van Wyk H, Roxburgh CSD, Horgan PG, Edwards J, et al. (2017) "Tumor invasiveness, the local and systemic environment and the basis of staging systems in colorectal cancer" Br J Cancer 116(11): 1444-1450.

5. Ziani L, Safta-Saadoun TB, Gourbeix J, Cavalcanti A, Robert C, et al. (2017) Melanoma-associated fibroblasts decrease tumor cell susceptibility to NK cell-mediated killing through matrix-metalloproteinases secretion. Oncotarget 8(12): 19780-19794.

6. Deryugina EI, Kiosses WB (2017) Intratumoral cancer cell intravasation can occur independent of invasion into the adjacent stroma. Cell Rep 19(3): 601-616.

7. Owusu BY, Galemmo R, Janetka J, Klampfer L (2017) Hepatocyte Growth Factor, a key timor-promoting factor in the tumor microenvironment. Cancers (Basel) 9(4)

8. Hu K, Miao L, Goodwin TJ, Li J, Liu Q, et al. (2017) Quercetin remodels the tumor microenvironment to improve the permeation, retention, and antitumor effects of nanoparticles. ACS Nano 11(5): 4916-4925.

9. Oh HJ, Bae JM, Wen XY, Cho NY, Kim JH, et al. (2017) Overexpression of POSTN in tumor stroma is a poor prognostic indicator of colorectal cancer. J Pathol Transl Med 51(3): 306-313.

10. Nystrom H, Jonsson M, Werner-Hartman L, Nilbert M, Carneiro A (2017) Hypoxia-inducible factor 1alpha predicts recurrence in highgrade soft tissue sarcoma of extremities and trunk wall. J Clin Pathol pic: jclinpath-2016-204149.

11. Tian B, Chen X, Zhang H, Li X, Wang J, et al. (2017) “Urokinase plasminogen activator secreted by cancer-associated fibroblasts induces tumor progression via PI3K/AKT and ERK signaling in oesophageal squamous cell carcinoma" Oncotarget 15857.

12. Ferrarelli LK (2017) Papers of note in Science Translational Medicine9 (384). Sci Signal 10(474).

13. Wang L, Wu H, Wang L, Zhang H, Lu J, et al. (2017) Asporin promotes pancreatic cancer cell invasion and migration by regulating the epithelial-to-mesenchymal transition (EMT) through both autocrine and paracrine mechanisms. Cancer Lett 398: 24-36.

14. Bravio S, Cadamuro M, Strazzabosco M, Fabris L (2017) Tumor reactive stroma in cholangiocarcinoma: the fuel behind cancer aggressiveness. World J Hepatol 9(9): 455-468.

15. Turunen SP, Tatti-Bugaeva O, Lehti K (2017) Membrane-type matrix metalloproteases as diverse effectors of cancer progression. Biochim Biophys Acta pii: S0167-4889(17)30087-30093.

16. Ishimoto T, Miyake K, Nandi T, Yashiro M, Onishi N, et al. (2017) Activation of Transforming Growth Factor Beta 1 signaling in gastric cancer-associated fibroblasts increases their motility, via expression of Rhomboid 5 Homolog 2, and ability to induce invasiveness of gastric cancer cells. Gastroenterology pic: S0016-5085(17)35398-353402.

17. Evan GI, Hah N, Littlewood TD, Sodir NM, Campos T, et al. (2017) Reengineering the pancreas tumor microenvironment : a 'Regenerative Program' hacked" Clin Cancer Res 23(7): 1647-1655. 
18. Busch S, Andersson D, Born E, Walsh C, Stahlberg A, et al. (2017) Cellular organization and molecular differentiation model of breast cancer-associated fibroblasts. Mol Cancer 16(1): 73.

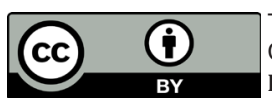

This work is licensed under Creative Commons Attribution 4.0 License

DOI: $10.19080 /$ CTOIJ.2017.05.555668
19. Wang Z, Tan Y, Yu W, Zheng S, Zhang S, et al. (2017) Small role with big impact: miRNAs as communicators in the cross-talk between cancerassociated fibroblasts and cancer cells. Int J Biol Sci 13(3): 339-348,

\section{Your next submission with Juniper Publishers will reach you the below assets}

- Quality Editorial service

- Swift Peer Review

- Reprints availability

- E-prints Service

- Manuscript Podcast for convenient understanding

- Global attainment for your research

- Manuscript accessibility in different formats

( Pdf, E-pub, Full Text, Audio)

- Unceasing customer service

Track the below URL for one-step submission https://juniperpublishers.com/online-submission.php 